Ensembles during visual search in peripheral vision

1

18 Correspondence should be addressed to: Ömer Dağlar Tanrıkulu, Faculty of

19 Psychology, School of Health Sciences, University of Iceland, Reykjavik. Email:

20 daglar@hi.is

21 The data from the experiments reported in this paper and the scripts for statistical

22 analyses are available at https://osf.io/ckpyu/.
This manuscript is now published in the "Vision Research" https://doi.org/10.1016/j.visres.2021.07.012

\section{Encoding perceptual ensembles during visual search in peripheral vision}

\section{Ömer Dağlar Tanrıkulu ${ }^{1}$, Andrey Chetverikov ${ }^{2}$, and Arni Kristjánsson ${ }^{1,3}$}

1 Faculty of Psychology, School of Health Sciences, University of Iceland, Reykjavik, Iceland

2 Visual Computation Lab, Center for Cognitive Neuroimaging, Donders Institute for Brain, Cognition and Behavior, Nijmegen, The Netherlands

3 School of Psychology, National Research University Higher School of Economics 
Observers can learn complex statistical properties of visual ensembles, such as their probability distributions. Even though ensemble encoding is considered critical for peripheral vision, whether observers learn such distributions in the periphery has not been studied. Here, we used a visual search task in order to investigate how the shape of distractor distributions influences search performance and ensemble encoding in peripheral and central vision. Observers looked for an oddly oriented bar among distractors taken from either uniform or Gaussian orientation distributions with the same mean and range. The search arrays were either presented in the foveal or peripheral visual fields. The repetition and role reversal effects on search times revealed observers' internal model of distractor distributions. Our results showed that the shape of the distractor distribution influenced search times only in foveal, but not in peripheral search. However, role-reversal effects revealed that the shape of the distractor distribution could be encoded peripherally depending on the inter-item spacing in the search array. Our results suggest that while peripheral vision might rely heavily on summary statistical representation of feature distributions, it can also encode information about the distributions themselves. 
53 There are large differences in the processing of visual information between the central

54 and peripheral visual fields. This reflects the fact that a much higher percentage of

55 neural structures in both retina and primary visual cortex are devoted to the foveal region

56 (e.g. Curcio \& Allen, 1987; Daniel \& Whitteridge, 1961; Hubel \& Wiesel, 1974; Drasdo,

57 1977; see Jóhannesson, Tagu \& Kristjánsson, 2018 for review). Resolution, acuity and

58 contrast sensitivity are higher for foveal than peripheral vision (Anstis, 1974; Virsu \&

59 Rovamo, 1979). However, only a small central region of our visual field corresponds to

60 the fovea, whereas the bulk of it is processed peripherally. This means that a significant

61 amount of our daily visual perception depends on our peripheral vision. In order to have

62 a complete picture of our visual capabilities, it is crucial to understand how processing

63 differs between central and peripheral vision.

64 Apart from diminished resolution and acuity, one of the main reasons why visual

65 performance is degraded in peripheral vision is crowding, which occurs when similar

66 items (i.e. distractors, or "flankers") are present nearby a target item (Stuart \& Bruian,

67 1962; Bouma, 1970; Levi, Klein \& Aitsabaomo, 1985; Wilkinson, Wilson \& Ellemberg,

68 1997). Recent work on crowding has suggested that peripheral visual information is

69 encoded as visual ensembles, which are represented in terms of summary statistics

70 (Levi, 2008; Pelli \& Tillman, 2008; Balas, Nakano, \& Rosenholtz, 2009; Rosenholtz,

71 Huang, Raj, Balas \& Ilie, 2012; Ehinger \& Rosenholtz 2016). This would enable

72 peripheral vision ${ }^{1}$ to process a large area of the visual field very quickly to detect any

73 potentially informative visual items or events. This would then guide consequent eye

74 movements made to project informative parts of the visual scene onto the fovea for

75 further processing. Therefore, examining how visual ensembles are encoded in

76 peripheral vision could increase our understanding of how the visual scene is processed.

77 While the visual system can represent a distribution of features (e.g. orientation, color,

78 size, facial expression) across many objects as ensembles, and can accurately and

79 efficiently extract the summary statistics of these ensembles (for reviews, see Haberman

80 \& Whitney, 2011; Alvarez, 2011; Whitney \& Leib 2018), foveal information is not needed

81 for this (Wolfe, Kosovicheva, Leib, Wood \& Whitney, 2015). Furthermore, it has even

82 been argued that summarizing information in the peripheral visual field as ensembles is

\footnotetext{
${ }^{1}$ Throughout this article, the term "peripheral" visual field is used to refer to all the areas outside of the fovea, which includes parafoveal and peripheral regions (i.e. might also be referred to as extra-foveal)
} 
83 an automatic compulsory process, which, in turn, explains why crowding becomes

84 stronger with increasing eccentricity (Parkes, Lund, Angelucci, Solomon, \& Morgan,

85 2001; Fischer \& Whitney, 2011; but see also Livne \& Sagi, 2007; Bulakowski, Post \&

86 Whitney 2011). While crowding can be seen as a limiting factor for object recognition in

87 the periphery, this compulsory averaging might facilitate scene perception as it has been

88 shown that peripheral processing is more useful for recognizing the gist of a scene than

89 foveal processing (Larson \& Loschky, 2009; Ehinger \& Rosenholtz, 2016).

90 Given this strong link between peripheral processing and ensemble perception, the

91 effects of eccentricity in encoding visual ensembles have not received the attention they

92 deserve. In particular, little work has focused on how ensemble encoding differs between

93 central and peripheral vision. Ji, Chen, \& Fu (2014) presented participants with sets of

94 faces where certain faces appeared in the foveal visual field, and others in the periphery.

95 Participants judged whether the overall emotion of the set was positive or negative. They

96 found that faces in the foveal region were given more weight than those in the periphery

97 in participants' judgments. On the other hand, by using gaze-contingent displays, Wolfe

98 et al. (2015) demonstrated that participants were equally accurate in reporting the mean

99 emotion of a set of faces when there was a foveal occluder and when there was not.

100 Both of these studies required explicit judgments about the mean value of a set of

101 features. These explicit judgments on statistical parameters of a feature distribution

102 might have limited power in revealing how accurately feature distributions in an

103 ensemble are encoded by the visual system. Recently, Chetverikov, Campana \&

104 Kristjánsson $(2016,2017 a, 2017 b, 2017 c)$ used a novel method to demonstrate that

105 observers can encode the probability density function underlying the distractor

106 distribution in an odd-one-out visual search task for orientation (2016, 2017a) and color

107 (2017b). Instead of using explicit judgments of distribution statistics, they measured

108 observers' visual search times varying target similarity to previously learned distractors,

109 which revealed observers' expectations of distractor feature distributions. This was

110 achieved by using role-reversal effects between the target and distractors, which occur

111 when feature values of target and distractors used on previous search trials are

112 swapped on the next trial (Kristjánsson \& Driver, 2008; Becker, 2010). This causes

113 search times to increase due to the similarity between the current target and the

114 previous distractors, compared to a case where current target and previous distractor

115 features are dissimilar. In this study, we use this implicit method and manipulate this 
116 similarity between the target and the previous distractors to assess whether observers

117 encode orientation distributions differently with central and peripheral vision. In this way,

118 we can examine observers' internal representations of ensembles, rather than their

119 explicit summary-statistics judgements that may in fact rely on these ensemble

120 representations.

121 Another goal of our study is to examine the effect of ensemble encoding on odd-one-out 122 visual search performance. Ensemble encoding can strongly influence visual search for 123 outliers (Cavanagh 2001; Alvarez, 2011; Whitney, Haberman \& Sweeny, 2014).

124 Detecting outliers among a set of items by comparing each item to all others would 125 require complex calculations. However, creating ensemble statistics of the relevant 126 feature distribution over the items would make outlier detection much simpler.

127 Rosenholtz et al. (2012) have shown that a model that represents the visual search area 128 by dividing it into patches and then analyzing these patches with respect to their 129 statistical summaries successfully predicts performance on classical visual search tasks.

130 When the difference between the statistical summary of the patch that includes both the 131 target and distractors and of the patch that only includes distractors increases, the 132 search becomes easier, even when the target-distractor confusability is the same at an 133 individual item level (e.g. search for 'O' among 'Q's vs. search for 'Q' among 'O's). In this 134 study, we will examine whether this influence of ensemble encoding on visual search 135 performance changes between the central and peripheral visual fields. More importantly, 136 previous studies have typically focused on the effect of target-distractor discriminability 137 (Duncan \& Humphreys, 1989, Palmer, Verghese \& Pavel, 2000), segmentability of 138 distractor features (Utochkin \& Yurevich, 2016; Cho \& Chong, 2019), or of summary 139 statistics (Rosenholtz et al. 2012) on visual search performance. In contrast, our 140 methodology allows us to investigate the effect of the shape of the distractor distribution 141 while keeping all those other factors as constant as possible.

142 In Experiment 1 we assessed observers' encoding of the orientation distribution of the 143 distractor set in an odd-one out visual search task, as well as its effect on search 144 performance. The search array was presented either in the central or the peripheral 145 visual field. In Experiment 2, we increased the spacing of the oriented bars as well as 146 their size in the peripheral condition, while decreasing their size in the central condition, 147 in order to make the two conditions more comparable in terms of processing capacity 148 and partly accounting for the cortical magnification factor. 
149 To summarize, our aim was to study search for targets among distractors with different

150 distributions to answer the question of performance differences between the periphery

151 and the center, as well as to measure feature distribution learning as described above

152 (and in Chetverikov et al., 2016; 2017a) as a function of retinal eccentricity.

\section{Experiment 1}

\section{Method}

155 Participants

156 Fourteen participants (seven males, age $M=32.14$ ) took part in the study. All had

157 normal or corrected-to-normal visual acuity. All participants signed an informed consent

158 form before participating. Four were paid for their participation, whereas the other ten,

159 who were staff or students at the University of Iceland, participated voluntarily. The

160 experiment was run in accordance with the Declaration of Helsinki and the requirements

161 of the ethics committee of the University of Iceland.

\section{Stimuli}

163 The display included a search array of 36 white lines arranged in a 6 by 6 invisible grid 164 extending to $9 \times 9^{\circ}$ on a grey background (Figure 1). For the central condition, the search 165 array was placed at the center of the screen, whereas for the peripheral condition it was 166 centred $9^{\circ}$ to the right or left of the center of the screen. The length of each line was $1^{\circ}$, 167 and their position in the grid was jittered by randomly adding a value between $\pm 0.25^{\circ}$ to 168 their horizontal and vertical coordinates. A red fixation dot with a radius of 5 pixels was 169 placed at the center of the screen.

170 Design

171 The details of the methodology we used in this study have been described in

172 Chetverikov, Hansmann-Roth, Tanrikulu \& Kristjánsson (2019). Here, we followed the 173 same design except for the addition of the peripheral condition and eye-tracking to make 174 sure that participants were fixating at the center of the screen. 
A.

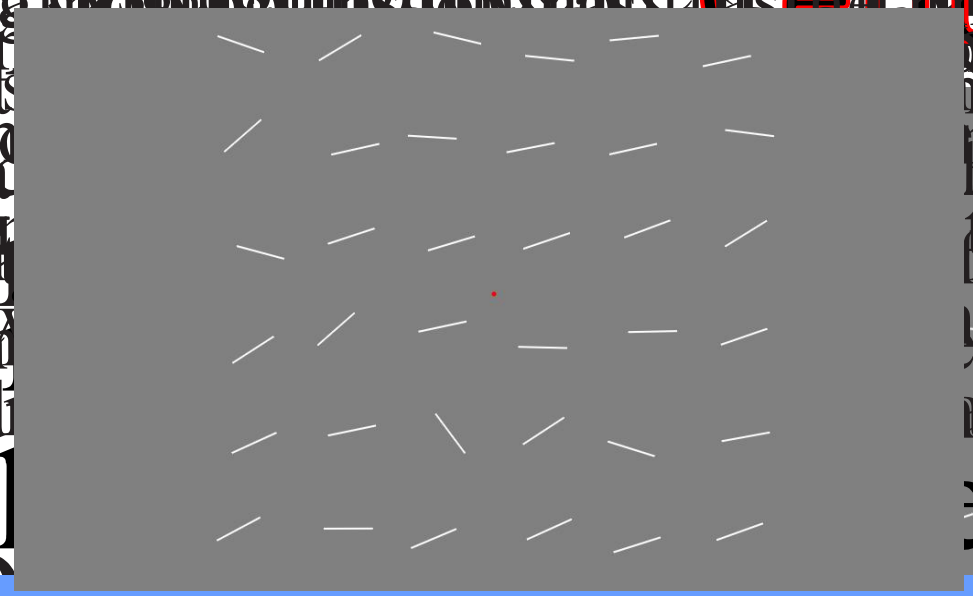

B.

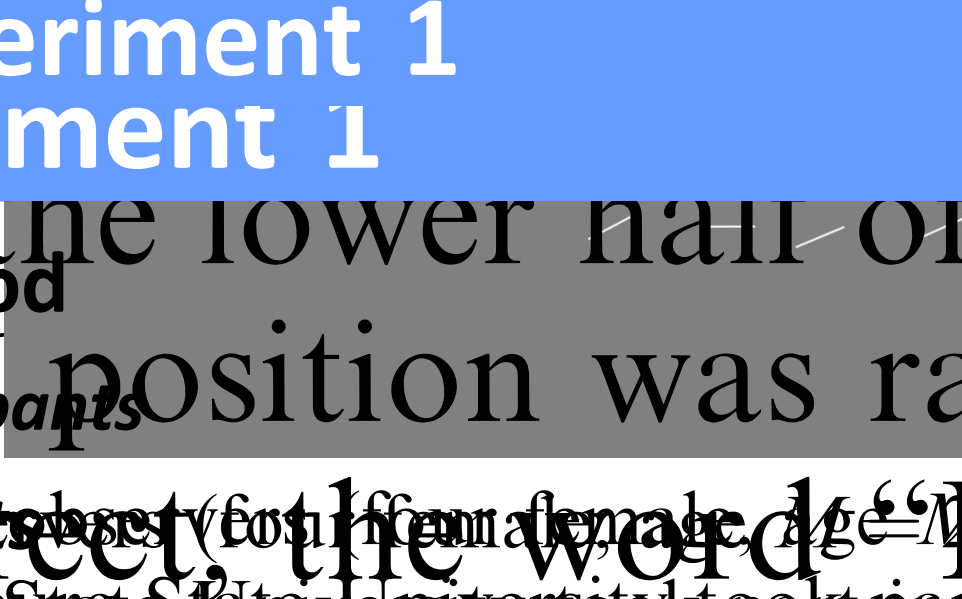

189 Figure 1: A. Example search array for the central condition in experiment 1. B. Example search array for the 190 peripheral condition in experiment 1 . In half of the peripheral trials, the search array was placed $9^{\circ}$ to the 191 right of center (red fixation dot) of the screen, and to the left of it in the other half.

193 The experiment included streaks of learning and test trials. Each learning streak

194 included five or six search trials in which the orientations of the distractors were sampled

195 either from a uniform (range $\left.=60^{\circ}\right)$ or a truncated Gaussian $\left(S D=15^{\circ}\right)$ distribution. The

196 Gaussian distribution was truncated at 2* $S D$ away from the mean so that the range for

197 the uniform and the Gaussian distribution was equal. This was additionally ensured by

198 setting the orientation of two distractor lines to the minimal and maximal values of a

199 given range and mean for both distributions. The mean orientation of the distractor

200 distribution was determined randomly for each learning streak and kept fixed during that

201 streak. The orientation of the target line was randomly determined with the restriction

202 that the target to mean-distractor distance in feature space was at least $60^{\circ}$. 
203 A single test trial followed the learning trials. The main variable that was manipulated in

204 the test trial was the distance between the target orientation on the current test trial and

205 the mean distractor orientation on the previous learning streak. This CT-PD (Current

206 Target - Previous Distractor) distance determines the extent of the role reversal effects.

207 In order to have a uniform distribution of CT-PD values, we divided the orientation space

208 into 12 bins where each bin covered a range of $15^{\circ}$. Then for each test trial, a CT-PD

209 value was randomly chosen from the bins ensuring that at the end of the experiment we

210 had equal numbers of CT-PD values from each bin. Given the chosen CT-PD value for

211 that test trial, the target orientation was determined to yield the chosen CT-PD distance

212 (Figure 2). The orientations of the distractors in the test trial were sampled from a

213 truncated Gaussian $\left(S D=10^{\circ}\right)$ distribution. The mean orientation of the distractors was

214 randomly determined with the restriction that the target to mean-distractor distance in

215 feature space was at least $60^{\circ}$.

216 Each participant completed 1872 search trials, which corresponds to a total of 288

217 (learning + test) streaks - i.e. 2 (search location: central or peripheral) $\times 2$ (distractor

218 distribution: Gaussian or uniform) $\times 12($ CT-PD bins) $\times 2$ (learning streak length: 5 or 6$) \times$

2193 (repetition).
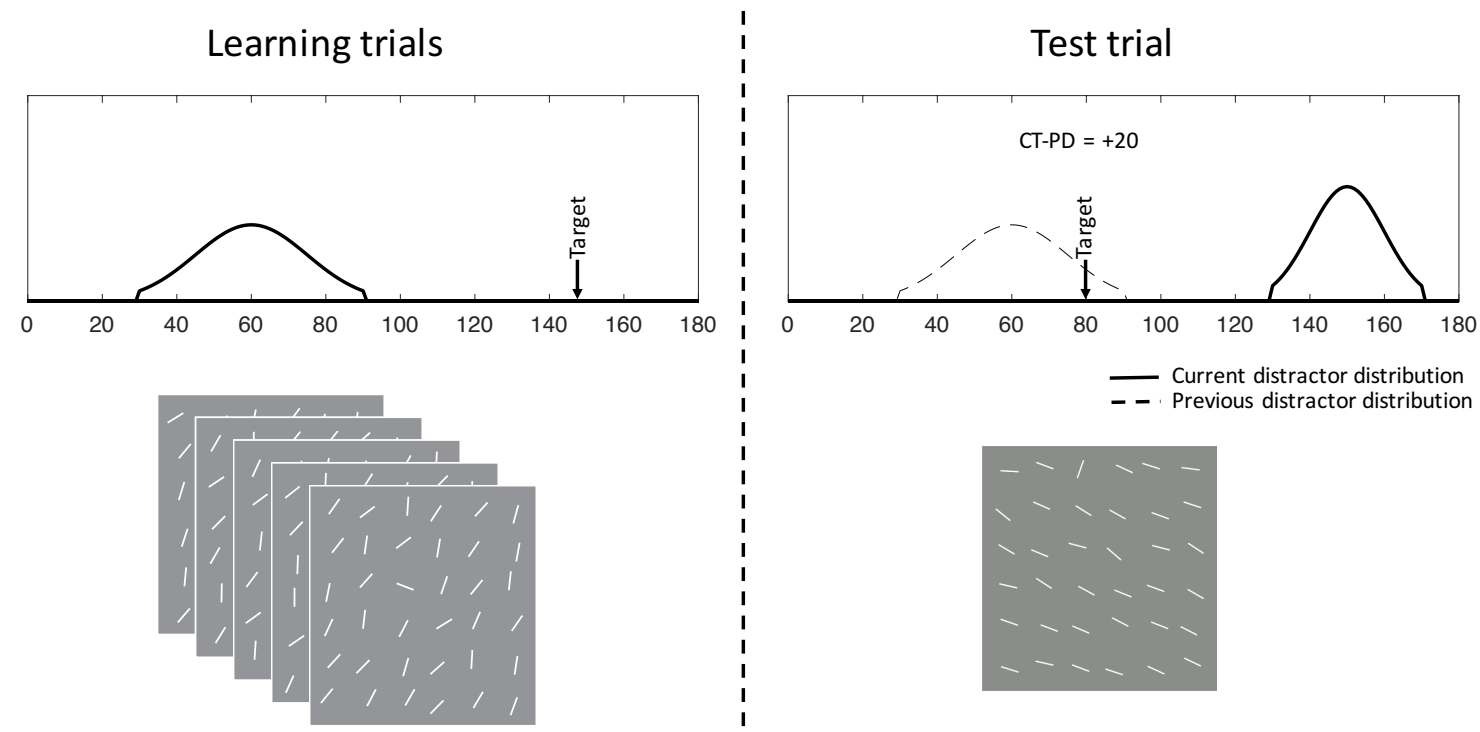

Figure 2: An example of a learning streak and a test trial. On the left, the distractor distribution and the target orientation is shown for a learning streak. The distractor orientations are sampled from the same distribution throughout the learning trials, however, the target orientation is randomly chosen given that it is at least $60^{\circ}$ away from the distractor distribution mean. On the test trial, the target orientation is chosen to yield different CT-PD values. In the example shown here, the distance between the current target orientation $\left(80^{\circ}\right)$ and the previous distractor distribution mean $\left(60^{\circ}\right)$ yields a CT-PD value of $+20^{\circ}$. 
228 Participants sat $100 \mathrm{~cm}$ away from a 24-inch LCD monitor $(1920 \times 1080)$ connected to a 229 Windows 7 PC. An EyeLink 1000 Plus eyetracker was used to track participants'

230 fixations. The experiment was presented using the Psychtoolbox (Brainard, 1997;

231 Kleiner, Brainard \& Pelli 2007) and EyeLink (Cornelissen, Peters \& Palmer, 2002)

232 toolbox extensions in MATLAB. We recorded eye movements from observers' right eye,

233 while their head was stabilized with a chin rest. The experiment included 8 blocks of

234 trials, and at the beginning of each block a 5-point calibration was performed. The

235 average calibration error for the participants was $0.37^{\circ}$

236 Participants were asked to find the oddly oriented line among the 36 oriented lines, and

237 indicate whether the oddly oriented line was in the upper three rows or in the bottom

238 three rows of the search array by pressing the up and down arrow keys accordingly.

239 Target position in the search array was randomized. Participants were asked to respond

240 as quickly and correctly as possible while keeping fixation on the red dot centred on the

241 screen. The next search trial was shown immediately after the participants responded,

242 except when an error was made. In that case, the word "Error" appeared in the middle of

243 the screen in a red font for $1 \mathrm{~s}$. In order to motivate subjects, a score was calculated for

244 each trial based on the participant's accuracy and response time (For correct response:

245 score $=10+(1-R T) * 10$; for errors: score $=-|10+(1-R T) * 10|-10$; where $\mathrm{RT}$ is

246 response time in seconds). The accumulated current score was shown to participants

247 during the breaks.

248 The experiment was completed in 8 blocks, split into 2 sessions. Each block consisted of

24936 learning + test streaks. Participants took as much resting time as they needed

250 between the blocks. At the beginning of each block, the participants were told in which

251 location on the screen the search array would appear (left, right or center) relative to the

252 fixation point. The location of the search array was kept fixed throughout a block, but

253 alternated across blocks. All conditions were counterbalanced, and trials were

254 randomized for each subject separately. The order in which participants performed trials

255 with different search array locations was also counterbalanced across subjects. For all

256 fourteen participants, the first session included 72 practice streaks (roughly 400 search

257 trials, where half of them in the central, other half in a peripheral location), which was

258 followed by three blocks of experimental trials. The second session included 24 practice 
259 streaks (i.e. 130 search trials) and five experimental blocks. The four participants who

260 were inexperienced with the search task were given a full practice session (i.e. 5 blocks

261 which corresponds to 180 streaks) before they started the two experimental sessions.

262 This was done to acquaint these participants with the search task, since their response

263 times were exceptionally high ( $>3 \mathrm{~s}$ ) when they were first introduced to the task. The

264 other ten participants were already experienced with similar visual search tasks, and

265 since their overall search times were already lower than $1 \mathrm{~s}$ they did not perform

266 separate practice sessions (but they still completed the practice trials embedded in the

267 experimental sessions). On average, each session took $50 \mathrm{~min}$. to complete.

269 Results and Discussion

270 In the central condition, trials where participants made an eye-movement outside of the 271 search array grid were excluded. In the peripheral condition the trials where participants 272 made an eye movement into the search area were excluded (overall $10 \%$ of all trials). If 273 such an eye movement occurs during the learning streak, the learning process can 274 potentially be interrupted. That is why we used longer learning streaks with five or six 275 trials, rather than two trials, which has been found to be enough for observers to encode 276 the distractor distribution with this method (Chetverikov et al., 2017a). Therefore, even if 277 the learning streak is interrupted by such an eye movement, this would only shorten the 278 length of the effective learning streak, but there would still be enough learning trials on 279 average to encode the distractor distribution. Therefore, exclusion of test trials was 280 independent of exclusion of learning trials.

281 Trials where RTs were too high ( $>3 \mathrm{~s}$ ) or too low $(<150 \mathrm{~ms}$ ) were also excluded (less 282 than $0.1 \%$ of remaining trials). Trials where participants made an error were excluded 283 from RT analyses (9\% of the remaining trials). RTs were log-transformed for all 284 analyses.

\section{Visual search performance}

286 Figure 3 shows visual search performance in the different conditions. Participants were 287 fastest when the distractor orientations were sampled from the Gaussian with $S D=10^{\circ}$ $288(M=648 \mathrm{~ms}, S D=81 \mathrm{~ms})$, followed by the Gaussian with $S D=15^{\circ}(M=666 \mathrm{~ms}, S D=$ 
$98 \mathrm{~ms})$ and they were slowest when sampled from a Uniform distribution $(M=688 \mathrm{~ms}$, $S D=118 \mathrm{~ms}$ ). A two-way repeated measures ANOVA yielded a significant effect of distribution type on $\mathrm{RT}\left(F(2,26)=10.91, p<0.001, \eta_{G}^{2}=0.02\right)$, as well as a significant

292 interaction between distribution type and search location $\left(F(2,26)=3.87, p<0.05, \eta_{G}^{2}=\right.$

293 0.003). However, search location alone did not have a significant effect on RTs $(F(1,13)$

$294=0.81, p>0.05)$. When the two search locations were analysed separately, an effect of

295 distribution type was only observed in the central condition $(F(2,26)=12.71, p<$

$\left.2960.05, \eta_{G}^{2}=0.03\right)$., while in the peripheral condition distribution type had little influence on

$297 \operatorname{RTs}(F(2,26)=3.35, p>0.05)$.

298 Participants were most accurate when the distractor orientations were sampled from the 299 Gaussian with $S D=10^{\circ}(M=0.95, S D=0.02)$, followed by the Gaussian with $S D=15^{\circ}$

$300(M=0.91, S D=0.03)$ and they were least accurate when sampled from a Uniform

301 distribution $(M=0.89, S D=0.04)$. A two-way repeated measured ANOVA revealed a

302 significant effect of distribution type on accuracy. $\left(F(2,26)=54.15, p<0.001, \eta_{G}^{2}=0.37\right)$.

303 There was no significant main effect of location $(F(1,13)=0.26, p>0.05)$, nor an

304 interaction between the two factors $(F(2,26)=2.28, p>0.05)$.
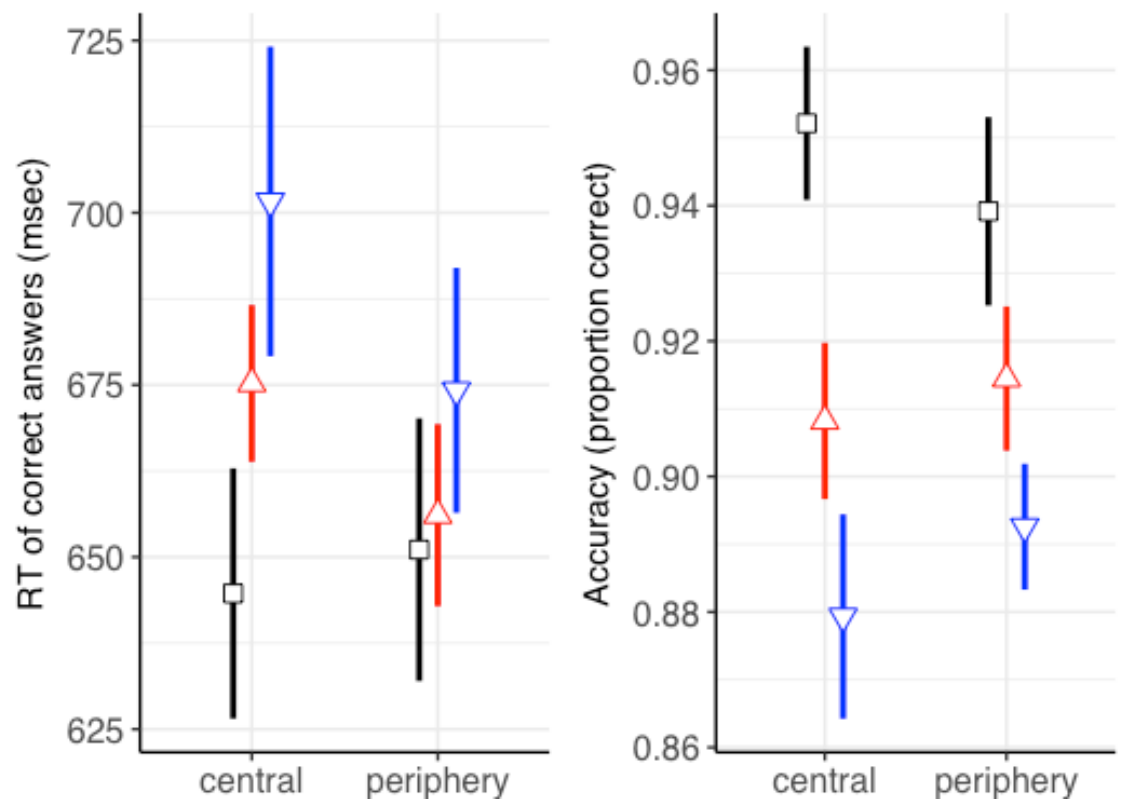

Distribution type

中 Gaussian, SD = 10

$\triangle$ Gaussian, SD $=15$

$\nabla$ Uniform

Location of search array

Figure 3: Visual search performance in Experiment 1. Error bars represent 95\% confidence intervals. 
310 Overall, search was faster and more accurate when the variance of the distractor

311 distribution was lower. While this finding is in line with previous studies, there was one

312 interesting exception to this general trend. When the search array was presented in the

313 periphery, the distractor distribution did not have a significant effect on search times,

314 even though the accuracy was similar for the central and peripheral conditions.

\section{Encoding of distractor distributions}

316 Figure 4 shows the CT-PD curves (response times on the test trials as a function of CT-

317 PD distance), which reflect the effect of role reversals between target and distractor

318 orientations. Since the distributions we used were symmetric, absolute values of CT-PD

319 distances were used in the following plots and analyses. Test trials following an error

320 were excluded from the analyses, because participants tend to slow down on a trial

321 following an error, which overrides the role-reversal effects.

322 In order to assess to what extent participants encoded the distractor distribution, we

323 compared these CT-PD curves to the corresponding type of the distractor distribution

324 used in the learning streak. Given that absolute values of CT-PD were used here,

325 following a learning streak in which distractors were sampled from a Gaussian

326 distribution, it would be expected that the search times in test trials would monotonously

327 decrease with increasing CT-PD values. Whereas, for the test trial following a learning

328 streak with uniform distribution of distractors with a range of $60^{\circ}$, the search times would

329 be expected to be roughly constant within the $30^{\circ}$ range, but then decrease outside of

330 that range. 


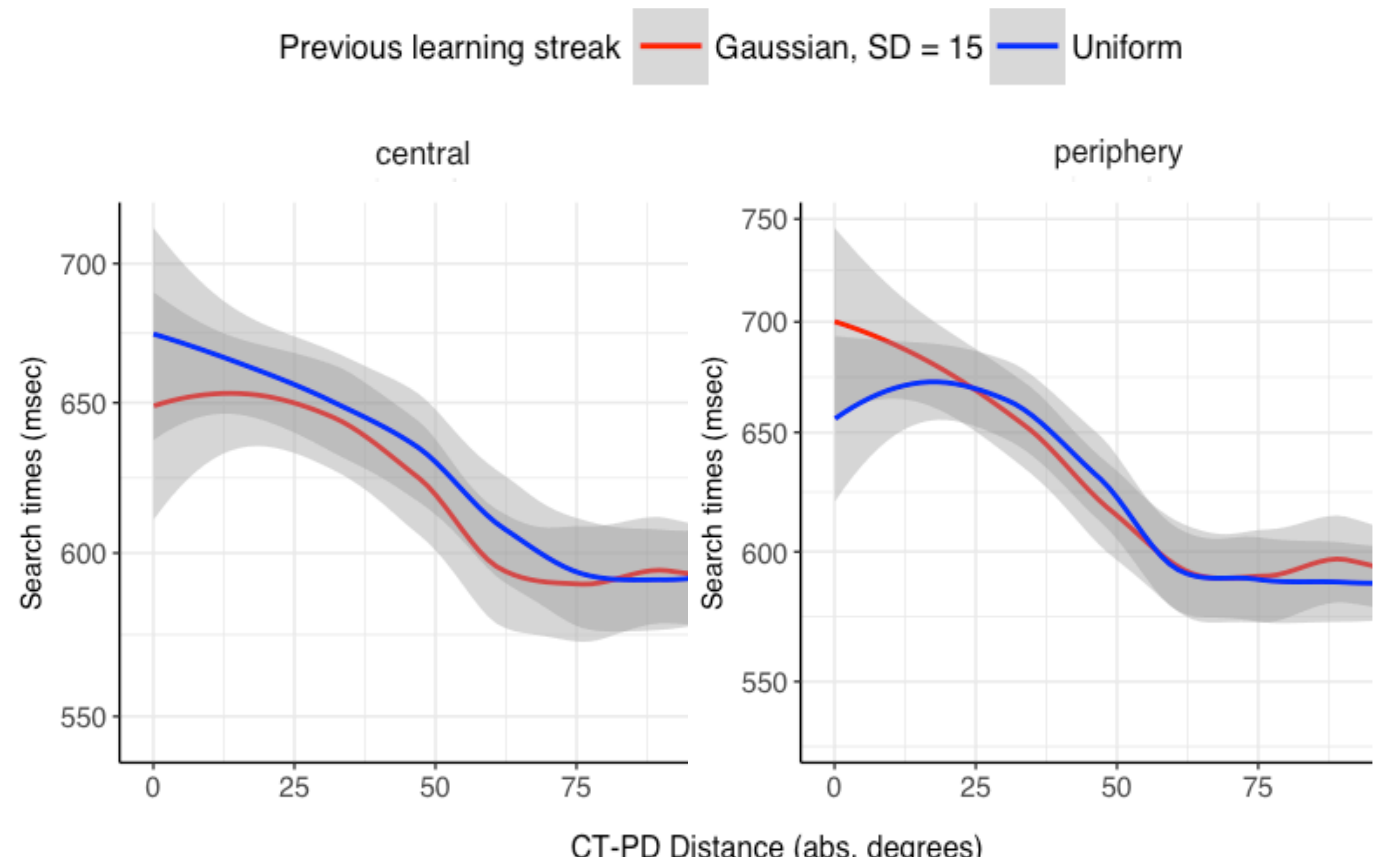

Figure 4: Role reversal effects in Experiment 1. Search times on test trials are plotted as a function of the distance (in orientation space) between the current target orientation in the test trials and the mean distractor orientation of the preceding learning streak (CT-PD: Current Target - Previous Distractor). Shaded area indicates $95 \%$ confidence intervals of the local (loess) regression fit.

337 To test this, we fit several possible distribution models to the CT-PD curves we obtained

338 from the test trials (Figure 5). These models included a null model (i.e. constant RT over

339 CT-PD values), a linear model (i.e. RT linearly depends on CT-PD), a half-Gaussian

340 model (i.e. RT as a function of CT-PD described by a half-Gaussian with $S D=15^{\circ}$ ), a

341 uniform model (i.e. constant RT but with different baselines within and outside of the $30^{\circ}$

342 CT-PD range) and a uniform-with-decrease model (i.e. constant RT within the $30^{\circ} \mathrm{CT}$ -

343 PD range, then linear decrease outside of that range). More formal descriptions of the

344 models used in these analyses can be found in Chetverikov et. al. (2017b). Table 1

345 shows the fit quality for the two best models among all models for each search array

346 location, with accompanying Bayesian Information Criteria (BIC) of relevant model

347 comparisons. 
Ensembles during visual search in peripheral vision

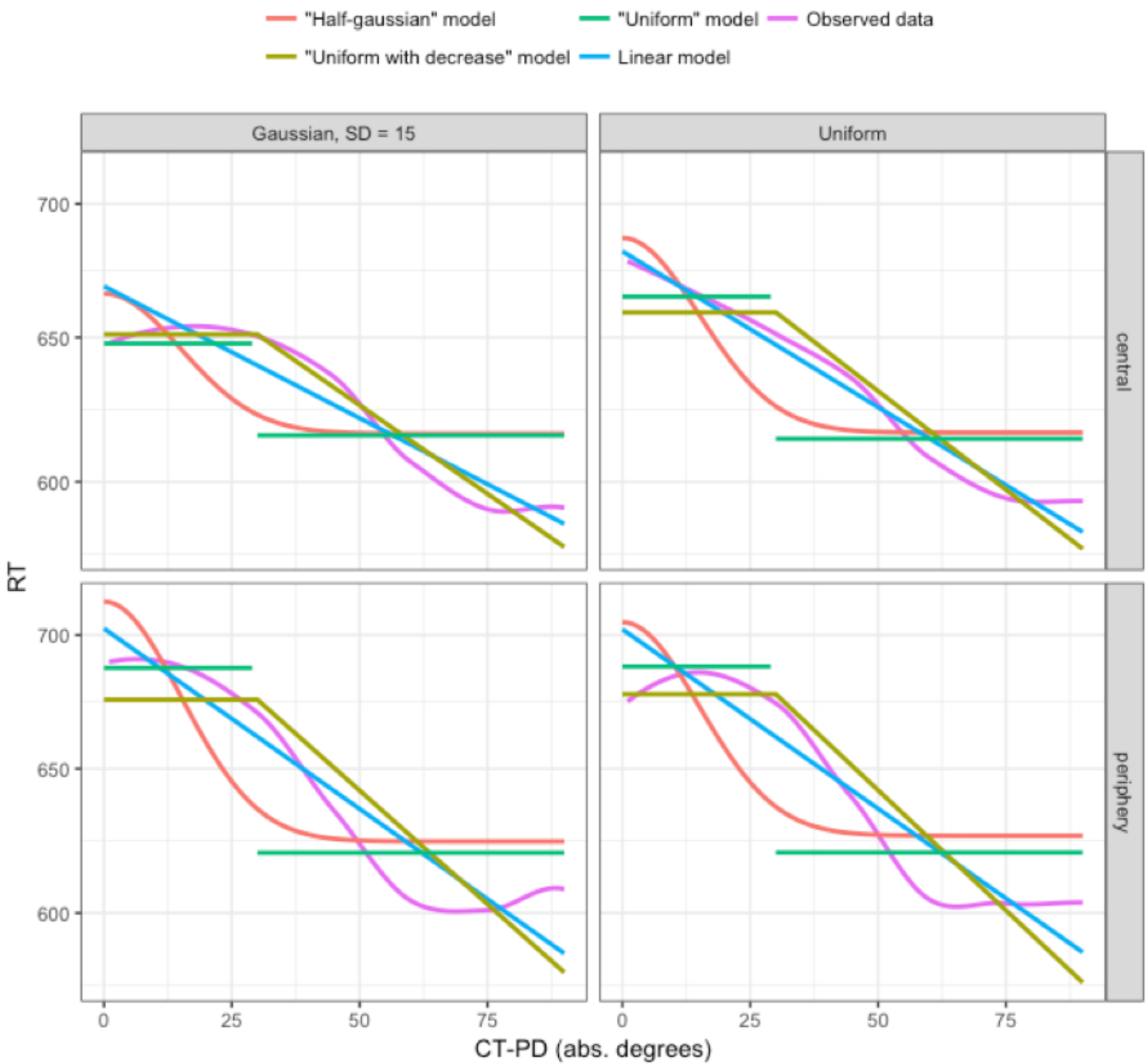

Figure 5: Fitted models and the CT-PD curves obtained from the test trial RTs in Experiment 1

Table 1: Model fits to the search times as a function of CT-PD distances in Experiment 1.

CENTER

PERIPHERY

Distractor Distribution

Best Model

$2^{\text {nd }}$ Best Model

$\triangle B I C$ : Best vs. Null

$\Delta$ BIC: Best vs. $2^{\text {nd }}$

\begin{tabular}{cc|cc}
\hline Gaussian & Uniform & Gaussian & Uniform \\
\hline Uni. w/ decrease & Linear & Linear & Uni. w/ decrease \\
\hline Linear & Uni. w/ decrease & Uni. w/ decrease & Linear \\
\hline 10.42 & 17.86 & 27.21 & 30.79 \\
\hline 0.41 & 0.92 & 4.58 & 3.69 \\
\hline
\end{tabular}

Note: $\Delta \mathrm{BIC}>2$ indicates "positive" evidence, $\Delta \mathrm{BIC}>6$ indicates "strong" evidence, while $\Delta \mathrm{BIC}>10$ corresponds to "very strong" evidence (Kass \& Raftery, 1995). 
357 Uniform distractor distributions were considerably better $(\Delta B / C>10)$ than the null model.

358 However, the best fits for both conditions were not better than the second best fits $(\triangle B I C$

$359<1)$. This suggests that when the search array was in the center, participants were able

360 to encode certain summary statistics (e.g. mean orientation) of the distractor

361 distributions, but notably, they were not able to encode the shape of that distribution.

362 The two best models, uniform with decrease and linear, fit the CT-PD curves obtained

363 from the Gaussian or Uniform distractor distribution condition equally well.

364 When the search array was presented in the periphery, the two best fits were

365 considerably better than the null model $(\Delta B I C>25)$. Not only that, the best fit for the

366 Gaussian condition (linear model) was better than the second best fit (uniform with

367 decrease model $)(\triangle B I C=4.58)$. This indicates that when the distractor distribution was

368 Gaussian, the search times linearly decreased as CT-PD value increased. When the

369 distractor distribution was Uniform, the best fit (uniform with decrease model) was better

370 than the second best fit (linear model) $(\triangle B I C=3.69)$, meaning that the RTs stayed

371 roughly constant for a certain range but then decreased outside of that range. This

372 suggests that participants were able to encode more details about the distractor

373 distribution (such as shape) when the search array appeared in the periphery. However,

374 this was not observed to the same extent when the array appeared centrally.

375 Whether the distractor distribution was encoded beyond its summary statistics was

376 assessed by the demonstration of a statistically meaningful shape correspondence

377 between the CT-PD curves and the physical distractor distribution. However, due to

378 noise in the visual system, a representation of a distribution would be expected to

379 involve certain approximations. For example, comparison of observers' priors and

380 natural statistics reveals important similarities but not an exact match between the two

381 (Girshick, Landy \& Simoncelli, 2011). This can explain why the best fit for the Gaussian

382 distractor distribution was a linear model rather than a half-Gaussian model for the

383 periphery condition. Both the linear and the half-Gaussian model involve a strict ${ }^{2}$

384 decrease in RT as CT-PD increases, which was the expected shape feature of a CT-PD

385 curve obtained following a Gaussian distractor distribution. However, this strict decrease

386 is not expected following a Uniform distractor distribution. Accordingly, the best model

\footnotetext{
${ }^{2}$ We use "strictly decreasing" to refer to a function that is always decreasing and does not remain constant (i.e. cannot include a plateau). We use "monotonic decrease" to refer to a function that is always decreasing or remaining constant (i.e. can include a plateau).
} 
387 for the uniform distractors in the periphery condition was the "Uniform with decrease"

388 model, which does not involve a strict decrease in RT as CT-PD increases.

389 Item heterogeneity (for example, from variance) is known to influence judgments of

390 visual ensembles (e.g. Marchant, Simons \& de Fockert, 2013). In our experiment, there

391 was a slight variance difference between the Gaussian and the uniform distribution as

392 their ranges were equal. Notably, however, in a previous study using the same implicit

393 methodology we used here, Chetverikov et al. (2016) showed that the shape

394 correspondence between CT-PD curves and distractor distributions cannot be explained

395 by this variance difference.

\section{Experiment 2}

397 In Experiment 2, we changed the spacing between the oriented lines, as well as their

398 lengths. For the peripheral condition, we increased both the spacing between the lines

399 and their length. Since effects of crowding increase with increasing eccentricity, these

400 changes will partly compensate for crowding in the periphery, as well as for the cortical

401 magnification factor. In addition, we decreased both the spacing between the lines and

402 their length in the central condition. These changes ensured that all the lines of the

403 search array in the central condition fell within the foveal region. These changes made

404 the two search array location conditions more comparable in terms of general

405 processing capacity.

\section{Method}

407 Participants

408 Fourteen participants (seven males, age $M=29.93$ ) took part in the study. Ten had 409 previously participated in Experiment 1. All had normal or corrected-to-normal visual 410 acuity. All participants signed an informed consent form before participating. Four were 411 paid for their participation, whereas the other ten, who were staff or students at the 412 University of Iceland, participated voluntarily. The experiment was run in accordance

413 with the Declaration of Helsinki and the requirements of the ethics committee of the 414 University of Iceland.

\section{Stimuli and Procedure}


416 The search display was the same as in Experiment 1, except that the size of the search

417 field and of the oriented lines were scaled differently for the peripheral and central

418 conditions. In the central condition, the 36 oriented lines of the search array were

419 arranged in a grid extending only to $4^{\circ} \times 4^{\circ}$ and centred on the screen. The length of

420 each line was $0.5^{\circ}$ and their position was jittered randomly by adding a value between

$421 \pm 0.1^{\circ}$ to their horizontal and vertical coordinates. For the peripheral condition, the search

422 array extended $13^{\circ} \times 13^{\circ}$, and was placed $11^{\circ}$ to the right or left of the fixation point. In

423 this way, the distance between the fixation point and the oriented line that was closest to

424 the fixation point $\left(4.5^{\circ}\right)$ was the same in the peripheral conditions of Experiment 1 and 2.

425 The length of each line in the periphery condition was increased to $1.4^{\circ}$, and their

426 position was jittered randomly between $\pm 0.3^{\circ} \mathrm{A}$ red dot with a radius of 5 pixels was

427 again used as a fixation point.

428 The equipment, procedure and the design of the experiment were exactly the same as in

429 Experiment 1. A 5-point eye-tracking calibration was again performed before each

430 experimental block. Average calibration error was $0.39^{\circ}$.

\section{Results and Discussion}

432 In the central condition, trials in which participants made an eye movement outside of

433 the search array grid were excluded; whereas in the peripheral condition trials where

434 participants made an eye movement into the search array were excluded from the

435 analysis (overall $10 \%$ of all trials). Trials in which RTs were more than $3 \mathrm{~s}$, less than 150

$436 \mathrm{~ms}$, and where participants made an error were also excluded from the RT analyses

437 (11\% of the remaining trials). RTs were log-transformed for all analyses.

\section{$438 \quad$ Visual search performance}

439 Figure 6 shows the RT and search accuracy for each of the conditions. A two-way

440 repeated measures ANOVA yielded a significant effect of distractor distribution type on

$441 \operatorname{RT}\left(F(2,26)=10.5, p<0.001, \eta_{G}^{2}=0.03\right)$. Similar to the results of Experiment 1 ,

442 participants were fastest when the distractor distribution was Gaussian with $S D=10^{\circ}(M$

$443=613 \mathrm{~ms}, S D=50 \mathrm{~ms})$, followed by the Gaussian $S D=15^{\circ}(M=619 \mathrm{~ms}, S D=59 \mathrm{~ms})$,

444 and they were slowest when the distractor distribution was uniform $(M=644 \mathrm{~ms}, S D=$

$44577 \mathrm{~ms}$ ). Similar to what we observed in Experiment 1, there was no significant effect of

446 search array location on $\operatorname{RT}(F(1,13)=3.23, p>0.05)$, however there was a significant 
447 interaction between search location and distribution type $(F(2,26)=22.25, p<$

$\left.448 \quad 0.001, \eta_{G}^{2}=0.03\right)$.

449 A repeated measures ANOVA on accuracy yielded a significant effect of distribution type

$450\left(F(2,26)=22.74, p<0.001, \eta_{G}^{2}=0.22\right)$, as well as a significant effect of search location

$451\left(F(1,13)=6.86, p<0.05, \eta_{G}^{2}=0.07\right)$. Participants were most accurate when the

452 distractor distribution was Gaussian with $S D=10^{\circ}(M=0.94, S D=0.03)$, followed by

453 Gaussian with $S D=15^{\circ}(M=0.9, S D=0.03)$, and were least accurate with uniformly

454 distributed distractors $(M=0.89, S D=0.03)$. Overall, participants were more accurate in 455 the central condition $(M=0.91, S D=0.04)$, than the peripheral condition $(M=0.89, S D$ $456=0.04)$. There was no significant interaction between the two factors $(F(2,26)=2.13, p>$ 457 0.05).
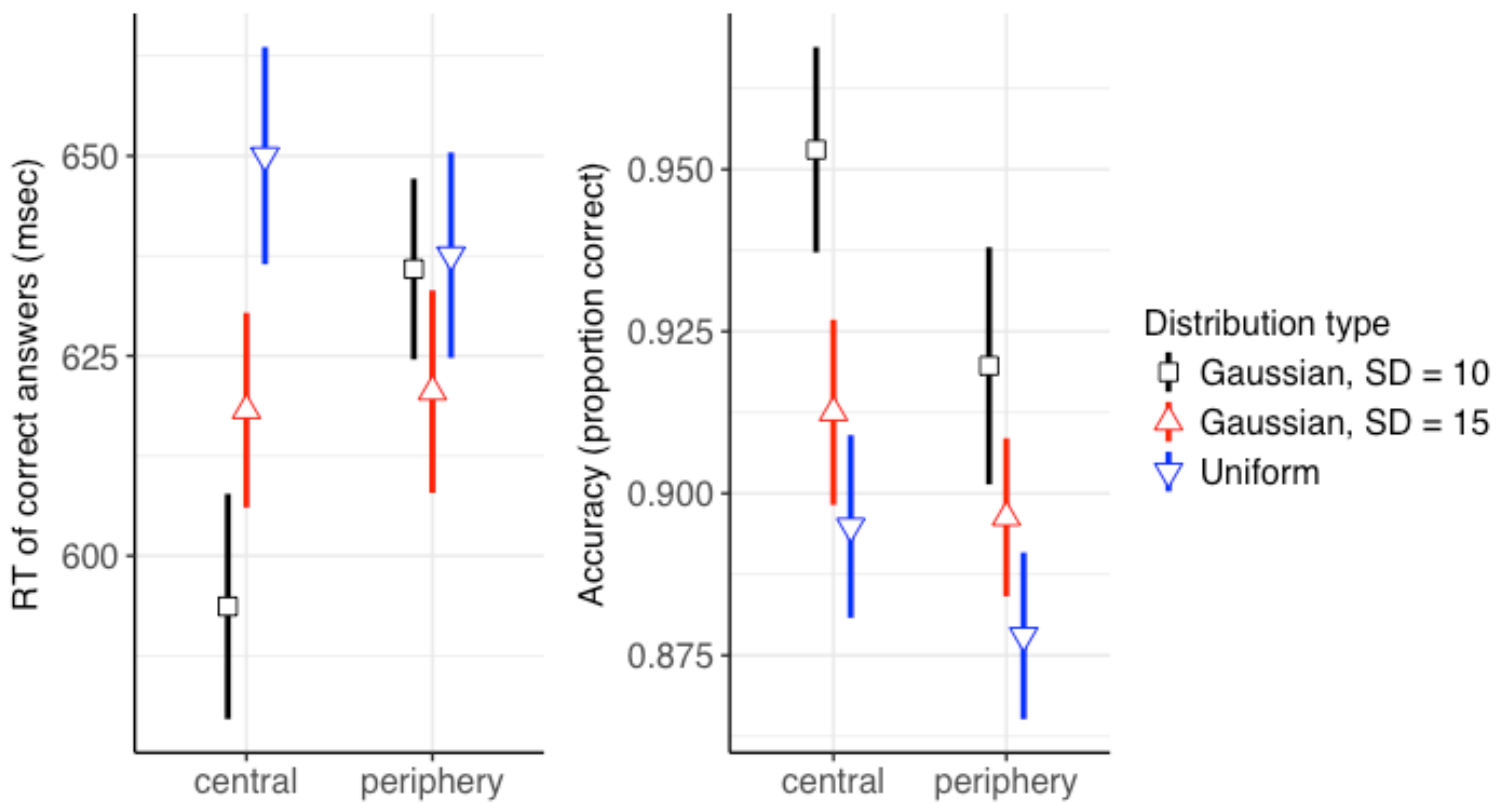

Location of search array

459 Figure 6: Visual search performance in Experiment 2. Error bars represent 95\% confidence intervals.

460 "Gaussian with $S D=10^{\circ "}$ (denoted by black) corresponds to the results obtained from the test trials, and the 461 other two distractor distribution types (Gaussian with $S D=15^{\circ}$ and uniform, which are denoted by red and 462 blue respectively) correspond to the results obtained from learning trials.

463 As in Experiment 1, participants in Experiment 2 were overall more accurate and faster 464 when the distractor distribution had lower variance. Effects of distribution type on search 465 times were, however, not observed in the peripheral condition. Overall average accuracy 466 was similar between the two experiments. Average RT in Experiment 2 was 43 ms lower 
467 than the one obtained in Experiment 1. This difference was likely due to those observers

468 in Experiment 2 who previously participated in Experiment 1 becoming slightly faster in

469 their response as they performed more sessions.

\section{Encoding of distractor distributions}

471 Figure 7 shows the CT-PD curves obtained from Experiment 2. The curves were

472 calculated in the same way as in Experiment 1. In the central condition, when the

473 distractor distribution was Gaussian, the search times monotonously decreased as CT-

474 PD increased. However, when the distractor distribution was uniform, search times

475 stayed roughly constant but then steadily decreased when the CT-PD exceeded the

476 range of the uniform distribution. These observations were confirmed by the model

477 comparisons done by fitting possible distribution models to RTs from the test trials

478 (Table 2 and Figure 8). The best fit for the Gaussian distractor distribution was the linear

479 model, whereas the best fit was the uniform-with-decrease model for the uniform

480 distractor distribution. Both of these best fits were better than the second best fit models

$481(\Delta B I C>2)$, and better than the null model $(\Delta B I C>8)$.

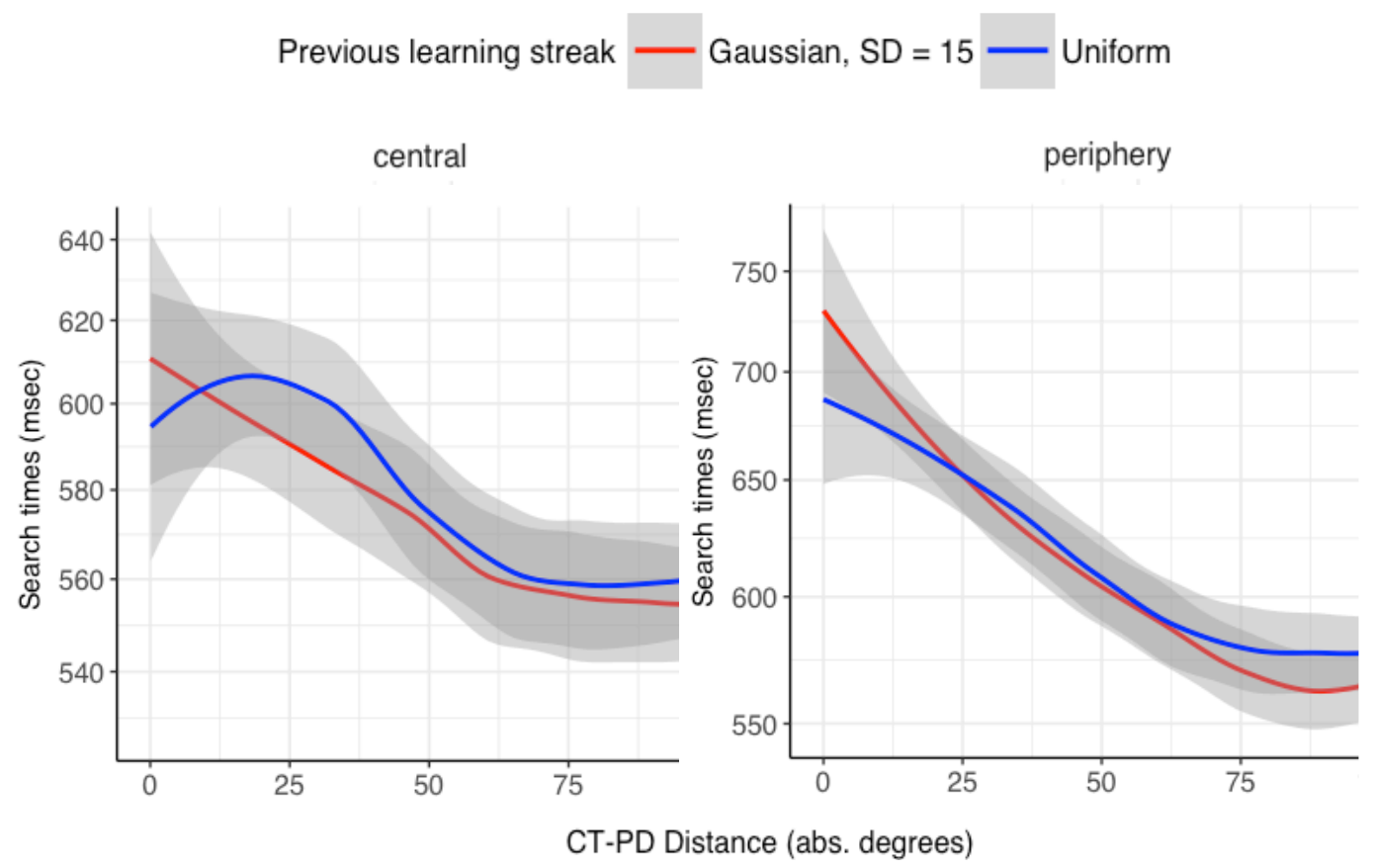

Figure 7: Role reversal effects in Experiment 2. Search times on test trials are plotted as a function of the distance (in orientation space) between the current target orientation in the test trials and the mean distractor orientation of the preceding learning streak (CT-PD: Current Target - Previous Distractor), separately for each search array location. Shaded area indicates $95 \%$ confidence intervals of the local

486

487 (loess) regression fit.

488 
Ensembles during visual search in peripheral vision

Table 2: Model fits to the search times as a function of CT-PD distances in Experiment 2.

CENTER

PERIPHERY

Distractor Distribution

Best Model

$2^{\text {nd }}$ Best Model

$\Delta B I C$ : Best vs. Null

$\Delta B I C:$ Best vs. $2^{\text {nd }}$

\begin{tabular}{cc|cc}
\hline Gaussian & Uniform & Gaussian & Uniform \\
\hline Linear & Uni. w/ decrease & Linear & Linear \\
\hline Uni. w/ decrease & Linear & Uni. w/ decrease & Uni. w/ decrease \\
\hline 8.92 & 11.41 & 65.00 & 34.11 \\
\hline 2.11 & 2.01 & 11.04 & 4.30 \\
\hline
\end{tabular}

Note: $\Delta \mathrm{BIC}>2$ indicates "positive" evidence, while $\Delta \mathrm{BIC}>6$ indicates "strong" evidence, whereas $\triangle \mathrm{BIC}>10$ corresponds to "very strong" evidence (Kass \& Raftery, 1995).

494

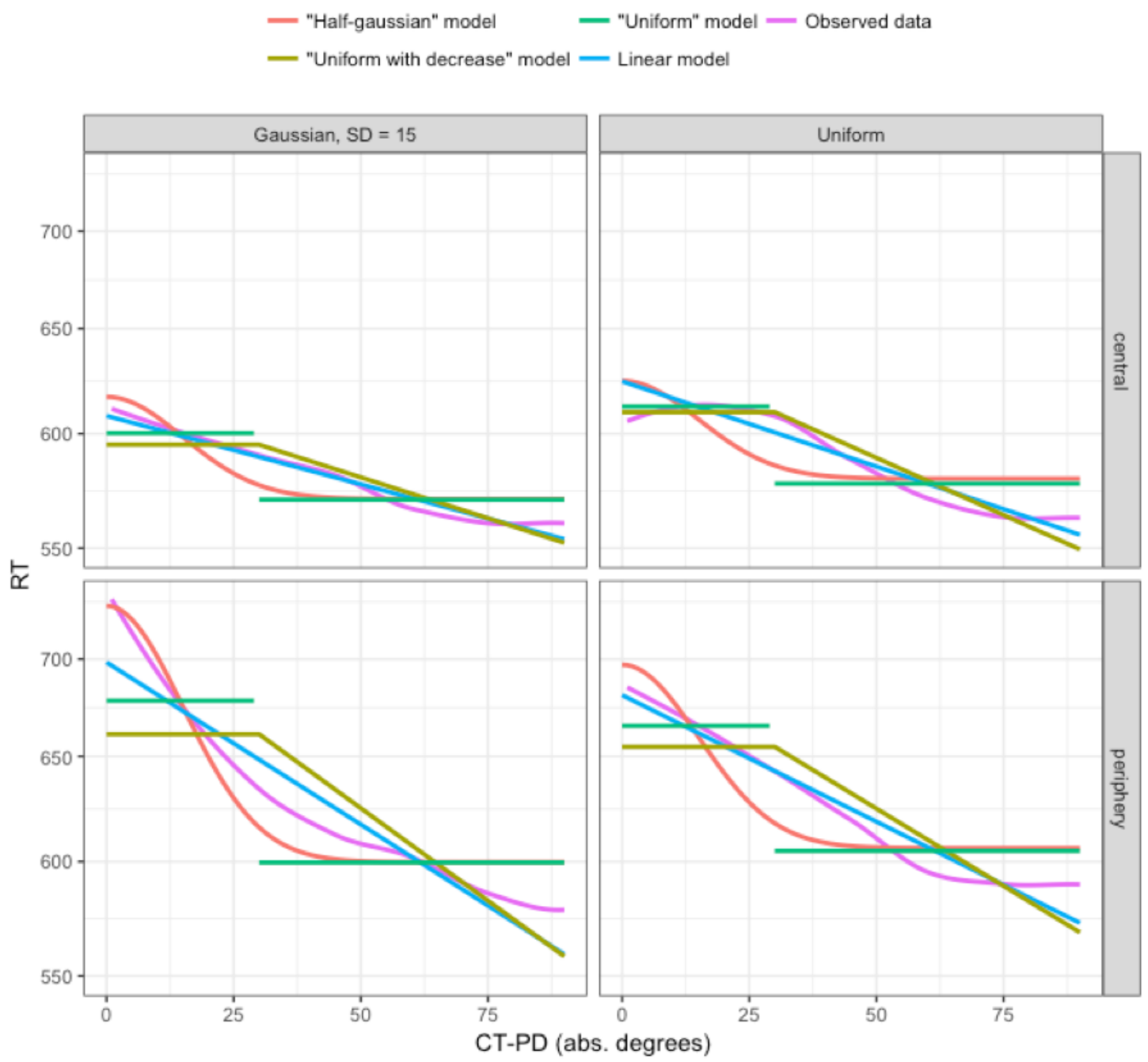

Figure 8: Fitted models and the CT-PD curves obtained from the test trial RTs in Experiment 2. 
498 For the peripheral condition, CT-PD curves did not change depending on the preceding 499 distractor distribution type. As shown on the right side of Figure 7, search times in the 500 periphery condition linearly decreased as CT-PD distance was increased, regardless of 501 the preceding distractor distribution type. This was also confirmed by the model fit 502 comparisons (Table 2). The best fit for both distractor distribution types was the linear 503 model, which had a considerably better fit than the second best models $(\triangle B / C>4)$, and 504 better than the null model $(\triangle B I C>34)$.

505 Contrary to what was observed in Experiment 1, participants in Experiment 2 were better 506 at encoding the shape of the distractor distribution when the search array was presented

507 in the center, compared to in the periphery. However, role reversals with CT-PD distance

508 around $0^{\circ}$ had a bigger influence on RTs in the periphery regardless of the preceding 509 distractor distribution. Response times decreased sharply and monotonously as CT-PD 510 increased, as seen from the high negative slopes obtained in the CT-PD curves of the 511 peripheral condition. For example, the estimated slope of the best fit model for the 512 Gaussian distractor distribution in the peripheral condition $(B=-0.03, S E=0.0003, z=$ $5138,67, p<.001$ ) was highly negative, whereas that level of steepness was not observed 514 for the best fit model for the Gaussian distractor distribution in the central condition $(B=$ $5150.01, S E=0.0003, z=-3,97, p<.001)^{3}$. This indicates that even though participants did 516 not encode the shape of the preceding distractor distribution in the periphery condition, it 517 is clear that the mean of the distractor distribution was specifically encoded.

\section{General Discussion}

519 In two experiments, we investigated similarities and differences in visual search

520 performance and ensemble encoding of distractor sets between the central and

521 peripheral visual fields. A crucial part of our experimental design involved investigating

522 the effects of distractor distributions with the same mean and range, but with different 523 shapes (Gaussian or uniform). Firstly, in both experiments, we found that the shape of

524 the distractor distribution strongly affected visual search times when the search was

525 performed in the foveal region, but we did not observe its effect in the periphery. In the

526 first experiment, more details of the shape of the distractor distribution were found to be

527 encoded in the peripheral condition than the central condition. In the second experiment,

\footnotetext{
${ }^{3}$ Note that analyses were done on log-transformed reaction times.
} 
528 we scaled down the search array for the central condition, but scaled it up for the

529 peripheral condition. This time, encoding of the shape of the distractor distributions was

530 found to be better in the central than the peripheral condition. Overall, we conclude that

531 in addition to summary statistics, detailed information (e.g. shape) about distractor

532 distributions can be encoded with peripheral vision, depending on the scale (or the inter-

533 item distance) of the search array.

534 In what follows, we speculate what these findings are likely to reflect.

\section{Search times in central vs. peripheral vision}

536 The influence of distractor distribution type on search times in the central condition of our

537 experiments is in line with previous studies. Distractor heterogeneity is known to have

538 negative effects on search performance (Duncan \& Humphrey, 1989; Rosenholtz, 2001).

539 In the central condition, the lower the variance of the distractor distribution, the faster

540 and more accurate participants were. Search times in the center differed even though

541 only the shape of the distractor distribution was changed while their summary statistics

542 were kept similar. However, this was not observed when the search array was presented

543 in the periphery. To our knowledge, this differential effect of distractor distribution on

544 search times in the center and periphery has not been observed before in the literature.

546 Eccentricity effects on search performance have been well established (Carrasco, Evert,

547 Chang \& Katz, 1995; Carrasco \& Yeshurun, 1998). Even though crowding increases with

548 increasing eccentricity, these eccentricity effects were found to be independent of

549 crowding (Carrasco \& Frieder, 1997; Madison, Lleras \& Buetti, 2018). However, other

550 researchers have also found effects of peripheral crowding on visual search

551 performance (Vlaskamp, Over \& Hooge, 2005). In our experiments, regardless of the

552 influence of eccentricity and crowding, the overall search times in the central and

553 peripheral conditions did not differ. This result was not surprising since the influences of

554 eccentricity and crowding are not as strong in pop-out search as in other types of visual

555 search tasks (Wertheim, Hooge, Krikke \& Johnson, 2006; Gheri, Morgan \& Solomon,

556 2007). In contrast with previous studies, however, we found that certain characteristics

557 of the distractor distribution had no influence on the search times in the periphery, even

558 though this influence was observed in foveal search. These characteristics include the

559 variance (or range) and the shape of the distractor distribution. This result was observed 
560 in both experiments, in other words, even when the spacing between the search items,

561 as well as their size, were manipulated to account for the cortical magnification factor.

562 Moreover, this difference between central and peripheral regions was observed even

563 though the overall search times and accuracy did not differ between the two regions.

564 We propose that this interaction between distractor distribution type and search array

565 location is consistent with the summary statistical account of visual search times of

566 Rosenholtz et al. (2012). According to this view, what determines search times is the

567 difference in summary statistics of a "feature pooling" region that contains the target

568 feature and a region that does not (i.e. only distractor features). The size of these

569 feature-pooling regions increases with eccentricity. This view can account for the search

570 times observed in our experiments. The summary statistics of the different distractor

571 distributions used in our study were very similar to each other. The mean of the

572 distractor distribution was equally distant (in orientation space) from the target on all

573 trials of the experiment. Guassian $S D=15^{\circ}$ and uniform distractor distributions had the

574 same range, which made their variance very similar. The only slightly different

575 distribution (with respect to its summary statistics) was the low variance for Gaussian $S D$

$576=10^{\circ}$. The reason why peripheral search times with the Gaussian $S D=10^{\circ}$ were not

577 lower than the other two distribution type conditions could be due to role reversal effects,

578 since the Gaussian $S D=10^{\circ}$ distribution was only used in the test trials. However, it

579 should be noted that the search times observed with Gaussian $S D=10^{\circ}$ in the central

580 condition were lower than the other two distribution conditions, even in Experiment 1,

581 where the overall amount of slowing due to role reversals was fairly similar in the center

582 and periphery.

584 In contrast to peripheral search, response times in the central condition were sensitive to

585 the different types of distractor distributions even though the differences between their

586 summary statistics were small. This contrast between foveal and peripheral search times

587 provides evidence for the view that summary statistical representations in peripheral

588 vision account for visual search times.

590 Ensemble coding with central vs. peripheral vision

592 Notably, our results go beyond the summary statistical account of peripheral vision. We

593 used the methods introduced by Chetverikov et al $(2016,2017)$ to asses any feature 
594 distribution learning in the periphery and in the center. Chetverikov et al. have shown

595 that observers can extract more detailed information about feature distributions within

596 visual ensembles than previous studies on ensemble perception have indicated. In

597 Experiment 1, when the search array was presented in the periphery, search times on

598 test trials with respect to CT-PD distance revealed that participants were able to encode

599 more than just the summary statistics of the distractor distribution. The shape of the CT-

600 PD curves observed on the test trials of Experiment 1 depended on the shape of the

601 distractor distribution used in the preceding learning trials. However, the effect of the

602 shape of the distractor distribution was not observed in the periphery when the spacing

603 of the search items was increased in Experiment 2. This observation is in line with other

604 studies demonstrating that crowding facilitates ensemble coding (Parkes et al., 2001;

605 Fischer \& Whitney, 2011). Since the peripheral search array was more crowded (low

606 inter-item spacing) in Experiment 1 than Experiment 2, this increased crowding might

607 have facilitated a more efficient process, perhaps akin to texture segmentation (Julesz,

608 1981), which, in turn, can explain why the shape of the distractor distribution was

609 encoded in more detail for the periphery condition in Experiment 1 but not Experiment 2.

611 However, in Experiment 2, when the target orientation on test trials was set to the mean

612 orientation of the distractor distribution on the preceding learning trials (i.e. CT-PD $=0$ ),

613 the effect of role reversal was stronger in the periphery than in the center (Figure 7). Not

614 only that, RTs decreased linearly with a relatively high negative slope as soon as CT-PD

615 increased. This suggests that the visual system relied heavily on the mean orientation of

616 the distractor distribution for finding the target in the periphery condition of Experiment 2,

617 rather than encoding other details of the distractor distribution.

619 What factors could have facilitated the encoding of information about the shape of the

620 distribution as opposed to its summary statistics? Apart from the effect of crowding

621 mentioned above, another reason could be the increase in inter-item spacing in

622 Experiment 2, which made a significant part of the search array appear at even higher

623 eccentricity. This could have made the encoding of the distractor distributions more

624 difficult. In fact, participants made more mistakes in the periphery condition than the

625 central condition in Experiment 2. This could force peripheral vision to rely on the

626 summary statistics of the distractor distribution, rather than encoding the distribution

627 itself. However, further empirical evidence is needed to confirm such an explanation. 
629 Overall, we suggest that the difference in results between the two experiments reflect

630 performance differences of the ensemble encoding mechanism in peripheral vision due

631 to the changes introduced to the visual stimuli, rather than two different encoding

632 mechanisms (e.g. summary statistics vs. distribution). In other words, we argue that the

633 peripheral visual system constructed a representation of the distractor distribution in both

634 experiments, however, this representation was less precise in Experiment 2, where more

635 weight was given to the mean of the distribution in building the representation of the

636 distribution, rather than its other features. Such an explanation is consistent with a

637 distribution-based framework of visual attention (see Chetverikov et al., 2017c for a

638 review).

640 The display used in the central condition in Experiment 1 was very similar to the ones 641 used in previous studies, which demonstrated that participants encode the shape of the 642 distractor distribution (Chetverikov et al. 2016, 2017a). However, in this study we did not 643 observe distribution shape learning in Experiment 1. The CT-PD curves obtained in this 644 study were noisier than those obtained in previous studies. One of the main differences 645 between our study and those previous ones was that we asked observers to maintain 646 fixation while they performed the search while in the previous studies participants were 647 free to move their eyes. Even though the size of the search array in Experiment 1 was 648 smaller than the ones used previously by Chetverikov et al. $(2016,2017 a)$, when 649 participants fixated at the center of the array, the lines at the outer part of the array still 650 fell outside of the foveal region. Some participants in our study reported that in the 651 central condition when they saw a candidate line that they thought was the target, they 652 sometimes struggled with inhibiting their eye movements towards that candidate line. It 653 is possible that this inhibition of eye movements influenced the encoding of the shape of 654 the distractor distribution. Our results from Experiment 2 support this interpretation 655 because when the search array was scaled down so that almost all the oriented lines fell 656 within $2^{\circ}$ around the fixation point, more details about the shape of the distractor 657 distribution were encoded. However, further studies are needed in order to understand 658 the influence of eye movements on the learning of distractor distribution shape.

660 The main difference between the two experiments was the scaling of the search array 661 and the difference in results might also be explained by the degree of mismatch between 
662 the size of the spatial filters in the periphery (or fovea) and the spatial resolution of the

663 search array. Such a mismatch can influence performance in texture segmentation

664 tasks. The task and the stimuli used in our study have parallels with texture

665 segmentation tasks. However, the effect of spatial resolution on texture segmentation

666 has been observed in explicit performance measures such as accuracy (Yeshurun \&

667 Carrasco, 1998). In our study, the pattern of results obtained for such performance

668 measures did not essentially differ between the two experiments. Therefore, if our

669 method had only relied on such explicit measures, then it could not have revealed any

670 differences in how scaling of the search array influences encoding of the distractor

671 feature distributions. This highlights an urgent need in the literature for implicit

672 experimental methods similar to the one used in this study. For example, future studies

673 can use our implicit method for novel insights into texture processing mechanisms,

674 eschewing the limitations of explicit experimental measures.

\section{Conclusion}

678 Our results showed that the shape and variance of the distractor distribution in a pop-out search for an oddly oriented line did not influence search performance when the search was performed with peripheral vision, whereas they had a significant effect when the search was performed around the foveal region. This result is in line with the view that visual information in the periphery is represented in terms of the summary statistics of

683 the scene. However, our results also showed that beyond summary statistics, more 684 details about the distractor distribution can be encoded with peripheral vision depending 685 on the spacing between the search items (or the overall size of the search array). When 686 the inter-item spacing was increased (in Experiment 2), participants tended to encode 687 the mean of the distractor distribution rather than details about its shape.

689 Our study also suggests that visual search studies of ensemble summary statistics 690 should be interpreted with caution. The visual search times were not sensitive to the 691 different distractor distributions when the summary statistics of those distributions were 692 roughly the same. This could suggest that peripheral vision relies mostly on summary 693 statistics. However, our implicit method revealed that, under certain conditions, 694 participants' peripheral vision encoded more detailed information about the distractor 695 distribution than only the summary statistics. These results call for the increased use of 
696 implicit experimental methods for a deeper understanding of what information is

697 encoded from visual ensembles.

References:

702 Alvarez, G. A. (2011). Representing multiple objects as an ensemble enhances visual

703 cognition. Trends in Cognitive Sciences, 15(3), 122-131. doi:10.1016/j.

704 tics.2011.01.003.

705 Anstis, S. M. (1974). Letter: A chart demonstrating variations in acuity with retinal

706 position. Vision Research, 14, 589-592.

707 Balas, B. J., Nakano, L., \& Rosenholtz, R. (2009). A summary-statistic representation in

708 peripheral vision explains visual crowding. Journal of Vision, 9(12):13, 1-18,

709 doi:10.1167/9.12.13.

710 Becker, S. I. (2010). The role of target-distractor relationships in guiding attention and 711 the eyes in visual search. Journal of Experimental Psychology: General, 139(2), 247712 265. http://dx.doi.org/10.1037/a0018808.

713 Bouma, H. (1970) Interaction effects in parafoveal letter recognition. Nature 226, 177714178.

715 Brainard D. (1997). The Psychophysics Toolbox. Spatial Vision, 10 (4), 433-436.

716 Bulakowski PF, Post RB \& Whitney D. (2011). Dissociating crowding from ensemble 717 percepts. Atten. Percept. Psychophys. 73(4):1003-9

718 Carrasco, M., Evert, D. L., Chang, I., \& Katz, S. M. (1995). The eccentricity effect: Target 719 eccentricity affects performance on conjunction searches. Perception \& Psychophysics, $720 \quad 57,1241-1261$.

721 Carrasco, M. \& Frieder, K. S. (1997). Cortical magnification neutralizes the eccentricity 722 effect in visual search. Vision Research, 37(1), 63-82.

723 Carrasco, M., \& Yeshurun, Y. (1998). The contribution of covert attention to the set-size 
724 and eccentricity effects in visual search. Journal of Experimental Psychology: Human

725 Perception and Performance, 24, 673-692.

726 Cavanagh, P. (2001) Seeing the forest but not the trees. Nat. Neurosci. 4, 673-674

727 Chetverikov, A., Campana, G., \& Kristjánsson, Á. (2016). Building ensemble

728 representations: How the shape of preceding distractor distributions affects visual

729 search. Psychological Science. http://dx.doi.org/10.1177/0956797617713787.

730 Chetverikov, A., Campana, G., \& Kristjánsson, Á. (2017a). Rapid learning of visual

731 ensembles. Journal of Vision, 17(21), 1-15. http://dx.doi.org/10.1167/17.2.21.

732 Chetverikov, A., Campana, G., \& Kristjánsson, Á. (2017b). Representing color

733 ensembles. Psychological Science. http://dx.doi.org/10.1177/0956797617713787.

734 Chetverikov A, Campana G, \& Kristjánsson Á (2017c) Learning features in a complex

735 and changing environment: $A$ distribution-based framework for visual attention and

736 vision in general, In: Progress in Brain Research, pp. 97-120 Elsevier.

737 DOI: 10.1016/bs.pbr.2017.07.001

738 Chetverikov, A., Hansmann-Roth, S., Tanrikulu, O.D. \& Kristjánsson, Á. (2019). Feature

739 Distribution Learning (FDL): a new method to study visual ensembles with priming of

740 attention shifts. Neuromethods, Springer

742 Cho, J., \& Chong, S. C. (2019). Search termination when the target is absent: The 743 prevalence of coarse processing and its intertrial influence. Journal of Experimental

744 Psychology: Human Perception and Performance, 45(11), 1455-1469.

745 Cornelissen, F.W., Peters. E., Palmer, J. (2002). The Eyelink Toolbox: Eye tracking with

746 MATLAB and the Psychophysics Toolbox. Behavior Research Methods, Instruments \&

747 Computers, 34, 613-617.

748 Curcio, C.A., Sloan, K.A, Packer, O., Hendrickson, A.E. \& Kalina, R.E (1987).

749 Distribution of cones in human and monkey retina: individual variability and radial

750 asymmetry. Science, 236(4801), 579-582.

751 Daniel, P. M., \& Whitteridge, W. (1961). The representation of the visual field on the 
752 cerebral cortex in monkeys. Journal of Physiology, 159, 203-221.

753 Drasdo, N. (1997) The neural representation of visual space. Nature (Lond.) 266, 554-

$754 \quad 556$

755 Duncan, J., \& Humphreys, G. W. (1989). Visual search and stimulus similarity.

756 Psychological Review, 96, 433-458.

757 Ehinger, K. A. \& Rosenholtz R. (2016) A general account of peripheral encoding also

758 predicts scene perception performance. Journal of Vision 16(2):13, 1-19

759 Fischer, J., \& Whitney, D. (2011). Object-level visual information gets through the

760 bottleneck of crowding. Journal of Neurophysiology, 106(3), 1389-1398.

761 doi:10.1152/jn.00904.2010.

762 Gheri, C., Morgan, M. J., \& Solomon, J. A. (2007). The relationship between search

763 efficiency and crowding. Perception, 36, 1779-1787.

764 Girshick, A.R., Landy, M.S., Simoncelli, E.P., 2011. Cardinal rules: visual orientation 765 perception reflects knowledge of environmental statistics. Nature Neuroscence. 14 (7),

766 926-932. http:// dx.doi.org/10.1038/nn.2831.

767 Haberman, J., \& Whitney, D. (2011). Ensemble perception: Summarizing the scene and 768 broadening the limits of visual processing. In J. Wolfe \& L. Robertson (Eds.), From

769 perception to consciousness: Searching with Anne Treisman. New York: Oxford

770 University Press.

771 Hubel, D. H., \& Wiesel, T. N. (1974). Uniformity of monkey striate cortex: A parallel

772 relationship between field size, scatter, and magnification factor. Journal of Comparative

773 Neurology, 158, 295-306.

774 Ji, L., Chen, W., and Fu, X. (2014). Different roles of foveal and extrafoveal vision in 775 ensemble representation for facial expressions. Engineering Psychology and Cognitive

776 Ergonomics Lecture Notes in Computer Science, 8532, 164-173.

777 Jóhannesson, ÓI, Tagu, J, \& Kristjánsson, Á. (2018) Asymmetries of the visual system 778 and their influence on visual performance and oculomotor dynamics. European Journal

779 of Neuroscience, 48: 3426-3445. https://doi.org/10.1111/ejn.14225 
780 Julesz, B. (1981). Textons, the elements of texture perception, and their interactions.

$781 \quad$ Nature, 290 (12), 91-97.

782 Kass, R. E., \& Raftery, A. E. (1995). Bayes factors. Journal of the American Statistical

783 Association, 90(430), 773-795. http://dx.doi.org/10.1080/01621459.1995. 10476572.

784 Kleiner M.,, Brainard D. H.,, Pelli D. G. (2007). What's new in Psychtoolbox-3?

785 Perception, 36, ECVP Supplement 14.

786 Kristjánsson, Á., \& Driver, J. (2008). Priming in visual search: Separating the effects of 787 target repetition, distractor repetition and role-reversal. Vision Research, 48(10), 1217788 1232. http://dx.doi.org/10.1016/j.visres.2008.02.007.

789 Larson A. M. Loschky L. C. (2009). The contributions of central versus peripheral vision

790 to scene gist recognition. Journal of Vision, 9(10):6, 1-16

791 Levi, D. M. (2008). Crowding: An essential bottleneck for object recognition: A mini792 review. Vision Research, 48, 635-654.

793 Levi, D., Klein, S. \& Aitsabaomo, A. P. (1985) Vernier acuity, crowding and cortical

794 magnification factor. Vision Research 25, 963-967.

795 Livne, T., \& Sagi, D. (2007). Configuration influence on crowding. Journal of Vision, $7967(2): 4,1-12$

797 Madison, A., Lleras, A. \& Buetti, S. (2018). The role of crowding in parallel search:

798 Peripherap pooling is not responsible for logarithmic efficiency in parallel search.

799 Attention, Perception \& Psychophysics 80(2), 352 - 373

800 Palmer, J., Verghese, P., \& Pavel, M. (2000). The psychophysics of visual search. Vision 801 Research, 40, 1227-1268.

802 Parkes, L., Lund, J., Angelucci, A., Solomon, J. A., \& Morgan, M. (2001). Compulsory

803 averaging of crowded orientation signals in human vision. Nature Neuroscience, 4, 739804744.

805 Pelli, D. G., \& Tillman, K. A. (2008). The uncrowded window of object recognition. Nature 806 Neuroscience, 11, 1129-1135. 
807 Rosenholtz, R. (2001). Visual search for orientation among heterogeneous distractors:

808 Experimental results and implications for signal-detection theory models of visual

809 search. Journal of Experimental Psychology: Human, Perception and Performance, 27, 810 985-999.

811 Rosenholtz, R., Huang, J., Raj, A., Balas, B. J., \& Ilie, L. (2012). A summary statistic

812 representation in peripheral vision explains visual search. Journal of Vision, 12(4), 1-17.

813 Stuart, J., \& Burian, H. (1962). A study of separation difficulty: Its relationship to visual

814 acuity in the normal amblyopic eye. American Journal of Ophthalmology, 53(471), 163-

815169.

816 Utochkin, I. S., \& Yurevich, M. A. (2016). Similarity and heterogeneity effects in visual

817 search are mediated by "segmentability". Journal of Experimental Psychology: Human

818 Perception and Performance, 42(7), 995-1007.

820 Virsu, V., \& Rovamo, J. (1979). Visual resolution, contrast sensitivity, and the cortical 821 magnification factor. Experimental Brain Research. 37,475-494.

822 Vlaskamp, B. N. S., Over, E. A. C., \& Hooge, I. T. C. (2005). Saccadic search

823 performance: The effect of element spacing. Experimental Brain Research, 167, 246824259.

825 Wertheim, A. H., Hooge, I. T. C., Krikke, K., \& Johnson, A. (2006). How important is 826 lateral masking in visual search. Experimental Brain Research, 170, 387-401.

827 Whitney D, Haberman J, Sweeny T. (2014). From textures to crowds: multiple levels of 828 summary statistical perception. In The New Visual Neuroscience, ed. JS Werner, LM 829 Chalupa, pp. 695-710. Cambridge, MA: MIT Press

830 Whitney, D., \& Leib, A. Y. (2018). Ensemble perception. Annual Review of Psychology, $831 \quad 69,12.1-12.25$.

832 Wilkinson, F., Wilson, H. R. \& Ellemberg, D. (1997) Lateral interactions in peripherally 833 viewed texture arrays. J Opt Soc Am A 14, 2057-68. 
Ensembles during visual search in peripheral vision

834 Wolfe, B. A., Kosovicheva, A. A., Leib, A. Y., Wood, K. \& Whitney, D. (2015) Foveal

835 input is not required for perception of crowd facial expression. Journal of Vision 15, 11.

836

837 Yeshurun, Y., \& Carrasco, M. (1998). Attention improves or impairs visual performance

838 by enhancing spatial resolution. Nature, 396(6706), 72.

839 